\title{
Papel del control instruccional en el estudio de las tendencias de regulación verbal*
}

Instructional Control Role in the Study of Verbal Regulatory Trends

Recibido: 20 de enero de 2015| Aceptado 25 de febrero de 2016

\author{
DYANNE Ruiz CASTAÑEDA** \\ MARIA INMACULADA GÓMEz BECERRA **** \\ Universidad de Almería, España
}

doi: 10.11144/Javeriana.upsy15-2.pcie

Para citar este artículo: Ruiz Castañeda, D. \& Gómez Becerra, M. I. (2016). Instructional Control Role in the Study of Verbal Regulatory Trends. Universitas Psychologica, 15(2), 135-152. http://dx.doi. org/Javeriana.upsy15-2.pcie

\footnotetext{
Artículo de investigación. Este trabajo no tuvo financiación alguna.

** Correo electrónico: dyanneruiz@hotmail.com

*** Departamento de Psicología. Correo electrónico: igomez@ual.es
}

\begin{abstract}
RES U MEN
La regulación verbal se ha estudiado ampliamente desde la teoría de los marcos relacionales por encontrarse en la base de múltiples fenómenos psicológicos, entre ellos, los procesos de aprendizaje en la infancia. El presente estudio tiene como objetivo principal evaluar si es más efectivo un procedimiento de aprendizaje con control instruccional (análogo experimental de la regulación verbal tipo pliance) versus un procedimiento sin control instruccional, esto es, sólo fortaleciendo las consecuencias directas (análogo experimental de la regulación verbal tipo tracking). Como objetivo secundario se pretende evaluar la efectividad de los tratamientos de manera diferencial según la tendencia inicial a seguir o no las instrucciones y evaluar su relación con variables implicadas en las diferencias individuales. Se utilizó un diseño de caso único A-B-C-A con réplicas y análisis entre sujetos. La muestra estuvo compuesta por nueve participantes (cinco chicos y cuatro chicas) con edades comprendidas entre 12 y 15 años. Los participantes realizaban una tarea de ordenador, en la que se evaluaba su tendencia a seguir o no las instrucciones, posteriormente realizaban dos bloques de entrenamiento, uno basado en el control instruccional y otro en moldeamiento por contingencias directas; finalmente se valoraba de nuevo su tendencia inicial. Adicionalmente, los participantes debían responder un cuestionario relativo a sus hábitos diarios. Los resultados mostraron que los participantes responden más rápido y con menor tasa de errores en el primer entrenamiento (control instruccional) y que variables como la rigidez, el autocontrol y el seguimiento instruccional podrían estar relacionadas con la facilidad en el aprendizaje de las mismas. Palabras clave

infancia; aprendizaje; tipo de regulación verbal; flexibilidad; control instruccional
\end{abstract}

\begin{abstract}
A B S T R A C T
The Verbal regulation has been studied extensively from Relational Frame Theory for being at the basis of many psychological phenomena, including learning processes in childhood. The present study has as main objective to assess if more effective learning procedure with instructional control (experimental analogue type regulation pliance) vs. without instructional control procedure, only strengthening the direct (regulation similar type experimental tracking). The secondary aim is to: assess the effectiveness of treatments differentially according to the initial tendency to follow or not the instructions and evaluate its relationship with variables involved in individual differences. We used a single case design with replicas and analysis ABCA between subjects. The sample consisted of 9 participants ( 5 boys and 4 girls) aged between 12 and 15 years. Participants performed a computer task in which assessed their tendency to follow or not the instructions, then performed two blocks of training, one based on the control in shaping instructional and other direct contingencies; finally back valued their tendency initial. Additionally, participants had to answer a test on your
\end{abstract}


daily habits. The results showed that participants respond faster and with less error rate in the first training (instructional control) and variables such as stiffness, self-control and instructional monitoring could be related to the ease in learning them.

Keywords

children; learning; verbal regulation; flexibility; instructional control

Conocer el repertorio de regulación verbal supone considerar las clases funcionales (Luciano \& Valdivia, 2012). Dichas clases funcionales son establecidas en las primeras etapas de la vida del niño y se definen como el conjunto de respuestas que pueden ser topográficamente (esto es, en su forma) diferentes, pero que quedan seleccionadas como funcionalmente equivalentes al acceder a la misma contingencia de reforzamiento (Luciano, Gómez, \& Valdivia, 2002). Así, los seres humanos desde la infancia se ven inmersos en un ambiente socioverbal en el que las palabras y otros estímulos adquieren funciones simbólicas, dándose la formación de preferencias condicionadas tempranas al igual que los reforzadores o preferencias primarias, por asociación directa o verbal a otros estímulos (algunos de ellos siendo estímulos ya condicionados), hacen que esos estímulos neutros cambien de reforzantes a aversivos y se altere la prioridad de unos sobre otros. Este proceso que se da durante la socialización del niño es denominado regulación verbal (Hayes, Brownstein, Zettle, Rosenfarb, \& Korn, 1986) y será el que permitirá al niño consolidar, o no, repertorios eficaces de autocontrol y que confluirá en la formación de los denominados estilos personales o patrones de personalidad.

La regulación verbal ha sido llamada de diferentes formas en la literatura del análisis del comportamiento, incluidas seguimiento de reglas, comportamiento gobernado por reglas y comportamiento instruccional (Luciano, Valdivia, \& Ruiz, 2012). En parte, debido a esta falta de consenso en la delimitación de la regulación verbal, se ha complejizado la definición de conceptos básicos, tal es el caso del término de regla ${ }^{1}$.Aquí es necesario resaltar que lo

1 Gómez (1996) realiza una exhaustiva revisión y síntesis de esta taxonomía. Así mismo, el lector interesado puede dirigirse a: Gómez, Moreno, \& López (2006) y a O'Hora \& Barnes-Holmes (2004). relevante debería ser la función de la regla (esto es, sus efectos sobre el oyente), por tanto se entenderá regla como estímulo que especifica contingencias (EEC) cuando controla una respuesta por primera vez, pero que, tras las primeras contingencias acaecidas en su presencia, sería más apropiado definirla como estímulo discriminativo (Sd) o como estímulo que altera la función de otros (Luciano, 1995).

De otro lado, Joyce \& Chase (1990) mencionan algunas de las características claves que diferencian la conducta gobernada por reglas y el moldeamiento por contingencias. En primer lugar, reglas y contingencias pueden ser diferentes en su manera de restringir la variabilidad de respuesta. Por ejemplo, la rápida adquisición de una respuesta gobernada por la regla relacionada a la respuesta, adquirida a través de la exposición a contingencias, indica que la historia preexperimental de refuerzo de los participantes siguiendo la regla es la responsable de un comportamiento similar al que se pide en la instrucción. En segundo lugar, las instrucciones combinan estímulos discriminativos elementales que pueden producir respuestas novedosas y complejas sin exposición previa a contingencias directas de la respuesta instruida. Por tanto, seguir una instrucción permite previamente establecer un repertorio sin una amplia exposición a la contingencia de refuerzo. Por el contrario, el comportamiento moldeado directamente por contingencias puede mostrar inicialmente un alto nivel de variabilidad y una exposición relativamente larga a contingencias que puede ser necesaria antes de que se seleccione un patrón de respuesta efectiva.

Desde una perspectiva contextual (Barnes-Holmes et al., 2001; Hayes, Barnes-Holmes, \& Roche, 2001; Hayes, Gifford, \& Hayes, 1998), se han diferenciado tres tipos diferentes de regulación verbal que conforman el proceso de socialización del niño y que se complejizan de manera progresiva, estos son pliance, tracking y augmenting. El pliance ocurre en función de una historia de consecuencias mediadas socialmente, mediadas por otros, al principio generalmente por los padres y luego por el mismo niño. Se da por la correspondencia entre la regla (ply) y la conducta que la sigue, pero sin que exista contacto con las contingencias directas del hacer; 
es decir, implicaría una historia de reforzamiento por el seguimiento de reglas per se, como clase de respuestas; por ejemplo, cuando la madre le dice al niño "termina tus tareas y te dejo salir al parque", al niño se le está incitando a hacerlo y cuando éste efectivamente termine su tarea, la consecuencia que dará la madre es dejarlo ir al parque recalcando que "el niño ha hecho lo que se le ha pedido que hiciera". Este tipo de regulación verbal debe ir reduciéndose paulatinamente en el niño a medida que crece para dar paso a otro tipo de regulación más vivencial; de no ser así, podría cronificarse y el niño sería mínimamente sensible a los cambios naturales de sus acciones. Por el contrario, el término tracking hace referencia al seguimiento de reglas sobre la base de una historia de correspondencia entre la regla (track) y las contingencias naturales (o rastreo de huellas naturales). Por tanto, esta clase de respuestas se conforma en función de una historia con múltiples circunstancias, en las que actuar de acuerdo a lo que se dice (lo especificado en la fórmula verbal, ya sea propia o ajena) ha sido reforzado por las consecuencias directas o naturales de dicha acción; por ejemplo, un niño escucha en el colegio que alguien dice "que hay un nuevo videojuego de moda y que todos deberían jugar”, el niño decide jugar a ese videojuego no porque alguien le haya sugerido que jugara, sino porque así podrá disfrutar de las consecuencias naturales de jugar con un nuevo videojuego. Por tanto, se genera un repertorio flexible y en buena parte independiente de las consecuencias mediadas por otros. En cualquier caso, ambos tipos de comportamientos gobernados por reglas quedarán incompletos si las contingencias que los mantienen no se relacionan verbalmente con los efectos a largo plazo. Por tal motivo, es necesario el tercer tipo de regulación, que sería el comportamiento tipo augmenting: regulación bajo el control de funciones transformadas de estímulo; sin embargo, este último no es objeto de estudio en este trabajo.

Una vez revisados los conceptos básicos de regulación verbal, y situados desde la perspectiva del comportamiento verbal, se puede definir lo que se reconoce en la literatura como (in) sensibilidad a unas $u$ otras contingencias. Dicho concepto hace referencia al fenómeno según el cual la regulación de la conducta por las consecuencias sociales (verbales) en formas de reglas puede dar lugar, bajo ciertas condiciones, a la atenuación o incluso a la eliminación total del efecto de las consecuencias directas sobre esa misma conducta (Gómez, Moreno, \& López 2006; Luciano, 1992b).

Específicamente, se genera una falta de ajuste a las contingencias directas, ya que la conducta quedaría bajo control de las palabras y sería sensible a éstas y no a aquellas. Por ende, no es que la conducta deje de ser sensible a las contingencias en general, sino a unas en particular porque pasa a ser sensible a otras (las derivadas de seguir la regla que son suministradas por la comunidad verbal) (Gómez, 1996). Un aspecto que sería clave para ubicar el tema de (in) sensibilidad a las contingencias y el control verbal, desde la perspectiva de la teoría de los marcos relacionales, es el hecho de que en el seguimiento de reglas, por su parte, lo relevante sería que la regla se actualice en los eventos que especifica a través de la transferencia de las funciones de estímulo, de acuerdo con las condiciones motivacionales del sujeto y provista por la historia de una persona a ciertas claves con contenidos verbales dispares (Luciano \& GómezMartín, 2001; Wilson \& Luciano, 2002). Este tipo de control verbal -discriminativo entre la regla y la acción-, que en ocasiones genera ajustes a las contingencias, muestra una insensibilidad hacia ellas como resultado de un desajuste de lo que pudiera ser efectivo para la persona. Algunos autores consideran que dicha insensibilidad es una característica del control instruccional, aunque otros autores como Galizio (1979, citado por Luciano, 1992a) consideran que la gente está más reforzada por seguir reglas que por otras cosas, es decir, más que una característica fundamental de la conducta verbal, sería un artefacto cultural.

Algunos de los problemas metodológicos que se han mencionado acerca de los estudios sobre regulación verbal son resumidos por Luciano (1992b) e incluyen: la falta de una tasa de respuesta estable antes de manipular las variables, utilizar un programa que permita un tipo de control diferente (diferente contacto con las contingencias discrepantes). 
Otro problema metodológico es la confusión entre las verbalizaciones sobre lo ya hecho y las verbalizaciones mientras se realiza la tarea experimental; por otra parte, también se añade el problema de las estrategias, ayudas y consecuencias utilizadas en relación al moldeamiento o instrucción de la conducta verbal, ya que debido a esto puede ser que la conducta que se cree moldeada, sea instruida. Por último, se señala como un aspecto importante la especificación de los repertorios verbales de los participantes cuando entran en contacto con las condiciones experimentales.

Diversos estudios han intentado mejorar los inconvenientes metodológicos mencionados, a la par de estudiar los diferentes procedimientos de control instruccional y de moldeamiento por contingencias. En este sentido, y estrechamente relacionado con el estudio que se presenta, se encuentra el trabajo de Dixon, Hayes, \& Aban (2000), quienes examinaron el comportamiento de toma de riesgo de los participantes en un contexto de juego patológico. En dicho estudio resaltan la importancia de la historia preexperimental de los participantes (uno de los aspectos metodológicos a tener en cuenta) cuando se intenta explicar la variabilidad experimental. Los resultados mostraron que variar los niveles de refuerzo no fue suficiente para llevar a cabo los cambios con respecto a cualquiera de estos comportamientos. Sugieren también que las propiedades que controlan tales reglas no se limitan a sus descripciones de las contingencias de refuerzo, sino también a las consecuencias sociales implícitas de seguir o no seguir las reglas. Además, se encontró que la presentación de reglas precisas era innecesaria para influir en la terminación del juego, o que los participantes en la condición de no reglas pueden haber estado desarrollando autoreglas precisas relacionadas con el juego, ya fuera de manera encubierta o manifestándolo.

Todo lo expuesto resalta la importancia del estudio de la regulación verbal y las diversas variables que pueden influir en ella. No obstante, aún son muchos los interrogantes que se plantean con respecto a si pueden ser mejores los procedimientos de aprendizaje que se realizan bajo el seguimiento de reglas o el moldeamiento por contingencias y qué relación podría tener en dichos procedimientos la historia de aprendizaje de los participantes.

Por otra parte, tal como proponen Luciano \& Valdivia (2012), la regulación de la conducta seleccionada en la historia personal podrá llegar a ser efectiva o destructiva dependiendo de si el valor de las consecuencias permite a la persona llevar la vida que le importa; así, desde la perspectiva funcional se entiende que la regulación destructiva es característica esencial de los trastornos de personalidad (TP). Dicha regulación destructiva se ha venido a denominar como trastorno de evitación experiencial -TEE-(Hayes, Wilson, Gifford, Follete, \& Strosahl, 1996) y es definido como un patrón inflexible de regulación que consiste en ajustar la conducta a la necesidad de escapar o no entrar en contacto con el malestar para, así, poder vivir; el problema se exacerba cuando este patrón de inflexibilidad se hace crónico o bien cuando ha sido el modo de funcionamiento instaurado desde la niñez o adolescencia, como es el caso de los TP.

Tal como se puede observar, fenómenos como el de flexibilidad vs. rigidez (perteneciente también al campo de las diferencias individuales) se encuentran estrechamente relacionados con la regulación verbal. De igual forma, una de las ventajas potenciales de tener un repertorio consolidado de seguimiento de reglas es el hecho de que los seres humanos aprenden a retrasar su respuesta y a hacer frente a los acontecimientos antes de que se pongan en contacto con estos; en otras palabras, las largas secuencias de comportamiento se pueden realizar y las consecuencias distantes en el tiempo y espacio, o extremadamente abstractas, pueden ser adoptadas, pero para lograrlo se requiere tener comportamientos de autocontrol (Törneke, Luciano, \& Valdivia, 2008). El fenómeno del autocontrol es otra de las variables que cobra gran importancia a la hora de estudiar la regulación verbal, este fenómeno implica hacer algo cuyas consecuencias inmediatas no son relevantes, e incluso pueden ser desagradables, con el fin de hacer algo relacionado verbalmente a la 
consecución de un resultado exitoso o valioso a más largo plazo (Gómez \& Luciano, 2000). Así, a medida que el niño aprende a responder a las formulas verbales y consolida su tendencia de regulación verbal, va generando repertorios eficaces, o no, de autocontrol, necesarios en situaciones en las cuales los niños deben mantenerse en una actividad repetitiva de la que no obtengan nada de inmediato, pero que les será útil en el futuro, aunque tengan otras opciones alternativas en las que implicarse; por ejemplo, preferir quedarse en casa a estudiar para un examen en vez de ver la televisión.

De acuerdo con lo expuesto, el presente estudio tiene como objetivo principal evaluar si es más efectivo un procedimiento de aprendizaje con control instruccional (por medio de un análogo experimental de la regulación verbal tipo pliance) versus un procedimiento sin control instruccional, sólo fortaleciendo las consecuencias directas (por medio de un análogo experimental de la regulación verbal tipo tracking). Los objetivos secundarios serían evaluar si esa efectividad de los tratamientos se muestra de manera diferencial según el tipo de tendencia inicial (pretest) de los participantes, ya sea la tendencia a seguir instrucciones o a ir en contra de ellas, y evaluar cuál es su relación con variables implicadas en el estudio de las diferencias individuales, tales como la rigidez y el autocontrol.

\section{Método}

\section{Participantes}

La muestra estuvo compuesta por nueve participantes (cinco chicos y cuatro chicas) con edades comprendidas entre 12 y 15 años $(M=12.4$, D.E $=0.88)$, que en el momento del estudio se encontraban escolarizados en dos centros educativos. La selección de los participantes se realizó en colaboración con sus profesores en base a los siguientes criterios: presentar un óptimo nivel de atención, mostrar habilidades básicas en el manejo de los ordenadores, tener adecuado conocimiento del idioma español y suficiente nivel de lectura y escritura.

\section{Materiales y contexto}

La tarea se llevó a cabo en aulas de los mismos colegios, aisladas de ruidos y distracciones, cada participante tenía una silla y una mesa con un ordenador. Los participantes debían realizar individualmente una tarea (juego) basada en un procedimiento de discriminación condicional creada por medio del programa Visual Basic que permitía ejecutar la tarea (instrucciones, estímulos, consecuencias, y registro de respuestas); dicho procedimiento permitía medir la tendencia de los participantes a seguir, o no, las instrucciones y entrenar a los participantes en el control instruccional y en el seguimiento de consecuencias directas.

Por otra parte, a los participantes se les administró un cuestionario creado por los investigadores (que actualmente se encuentra en proceso de validación estadística). Es un autoinforme denominado "Cuestionario sobre hábitos de vida" que consta de 20 ítems, divididos en cuatro escalas que miden: autocontrol (p.ej., "casi siempre que empiezo algo, no lo dejo hasta que lo terminó”), rigidez (p.ej., "una vez que he tomado una decisión, es difícil que cambie"), tendencia hacia el seguimiento de reglas (p.ej., "me siento bien cuando mis actividades diarias dependen de lo que otros deciden"), tendencia a comportamientos tipo tracking (p.ej., "soy curioso, me gusta conocer cosas nuevas"). El cuestionario es cumplimentado por el niño/adolescente teniendo en cuenta la manera en que éste se siente o comporta habitualmente y siguiendo una escala de tres alternativas de respuesta descritas como: «Si» (2), «A veces» (1), y «No» (0). La puntuación total se obtiene sumando las puntuaciones obtenidas en los 20 ítems, o si el investigador así lo requiere, puede obtener una puntuación de cada escala sumando los ítems pertenecientes a cada una de ellas. Los participantes después de diligenciar su código (asignado por el investigador para proteger la confidencialidad), su edad, su género y el curso escolar, recibían la siguiente instrucción: "las preguntas que vienen a continuación se refieren a la manera en que te comportas normalmente en tu VIDA DIARIA, contesta siempre pensando en la forma más habitual de comportarte. No existe 
una respuesta "correcta" para cada cuestión sino que todas las opciones son igualmente válidas. Tus respuestas serán totalmente confidenciales. Lee el enunciado y marca con un $\mathrm{X}$ en la casilla que creas que es más cercana a como tú eres".

\section{Diseño y variables}

Se utilizó un diseño de caso único A-B-C-A con réplicas y análisis entre participantes. Donde la variable dependiente era la tendencia de los participantes a seguir la instrucción o a seguir su propio criterio.

Las variables independientes eran la manipulación del tipo de entrenamiento:

V.I.1: entrenamiento basado en el control instruccional mediante un análogo experimental al comportamiento tipo pliance.

V.I.2: entrenamiento sin control instruccional mediante en un análogo experimental al comportamiento tipo tracking.

\section{Procedimiento}

La siguiente tabla resume el diseño y las fases del procedimiento:

En cuanto a las condiciones éticas del estudio, se pidió el consentimiento informado por medio de una carta a los padres de los participantes en donde se explicaba el objetivo del estudio, dicha carta fue enviada y recogida por medio de los profesores de curso. Una vez se contaba con el consentimiento, se acudía al aula regular de los participantes, momento en el que se les asignaba al azar un código, que no veía el investigador, que debían anotar en su agenda escolar con el fin de usarlo en el cuestionario (que se les pasaba a continuación) y en una tarea a la que acudirían otro día.

\section{TABLA 1.}

Diseño y fases del procedimiento
Pasada una semana, los participantes pasaban por parejas a un aula y se les asignaba un ordenador. Para empezar, uno de los investigadores daba las instrucciones generales, luego el participante debía seguir las instrucciones del ordenador: el programa pedía al participante registrar su código (aquel asignando previamente al azar y conocido solo por ellos con el fin de mantener la confidencialidad), su edad y género, y a continuación aparecía una presentación general de las normas del juego (véase figura 1).

Primera fase (línea base): medir la tendencia a seguir instrucciones

Esta primera fase tuvo como objetivo evaluar la tendencia de los participantes a seguir las instrucciones; esto es, si preferían seguir la instrucción que daba el ordenador o hacer lo que ellos determinaban o "intuían" como correcto. La tarea consistía en exponer al participante a una situación de discriminación condicional en la cual debía colocar dos peces en sus determinadas peceras. Las instrucciones específicas de cada fase eran dadas por el programa antes de iniciar dicha fase.

Esta primera fase estaba compuesta por un máximo de 25 ensayos, el participante debía completarlos todos para pasar a la siguiente (no había ensayos correctos o incorrectos ya que sólo se evaluaba su tendencia inicial y por esta razón no había refuerzo alguno). Cada ensayo consistía en introducir uno de los peces en la pecera que creía correcta arrastrándolo con el ratón, tal como se muestra en la figura 2; en un recuadro al lado derecho de la pantalla, aparecían dos opciones que podía seguir el sujeto a la hora de responder y que competían entre sí:

\begin{tabular}{llll}
\hline (A) Línea base & (B) V.I. 1 & (C) V.I. 2 & (A) Postest \\
\hline Evaluación de la & Entrenamiento basado en & $\begin{array}{l}\text { Entrenamiento sin control } \\
\text { instruccional (análogo }\end{array}$ & $\begin{array}{l}\text { Paradigma de } \\
\text { elección final }\end{array}$ \\
$\begin{array}{l}\text { expendencia inicial a } \\
\text { o a ir en contrucciones }\end{array}$ & $\begin{array}{l}\text { (análogo experimental al al comportamiento } \\
\text { tipo tracking) }\end{array}$ & $\begin{array}{l}\text { comportamiento tipo pliance) } \\
\text { tipos }\end{array}$ & \\
\hline
\end{tabular}

Fuente: elaboración propia 
En la parte superior aparecerán dos peces, uno a cada lado asi:
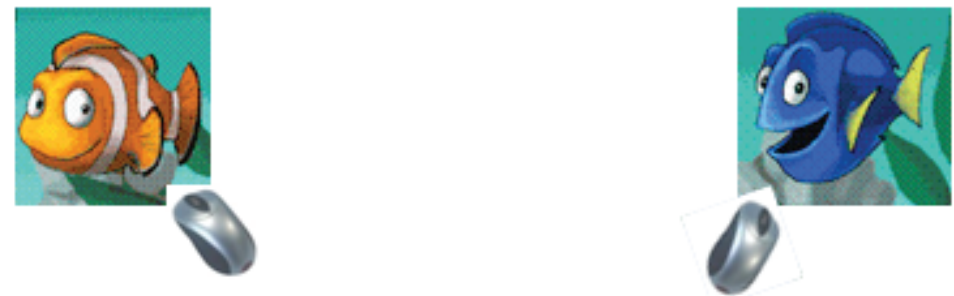

Elige el lugar que le corresponde, pinchando con el ratón uno de los peces y arrastrándolos hasta sus respectivas peceras que aparecen en la parte inferior. Luego debes hacer lo mismo con el otro pez.
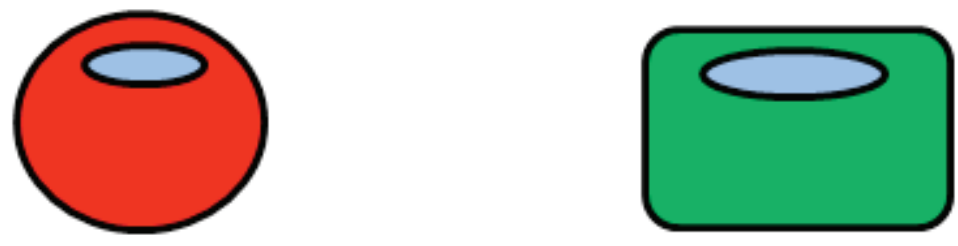

Figura 1. Ejemplo de las instrucciones iniciales del juego

Fuente: elaboración propia
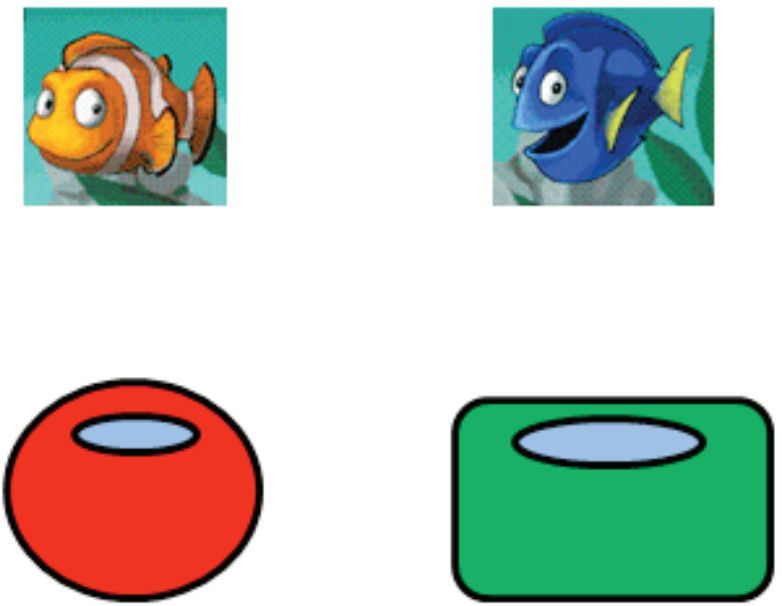

\section{Opción 1}

Debes

introducir el pez azul en la pecera cuadrada, y el naranja en la pecera circular

\section{Opción 2}

Seguirás tu propio criterio para hacer lo mejor posible.

Figura 2. Ejemplo de un ensayo en la fase de pretest

Fuente: elaboración propia 
-Opción 1, en donde el ordenador daba una instrucción específica sobre qué pez iba en cada pecera (por ejemplo: "debes introducir el pez azul en la pecera cuadrada y el naranja en la pecera circular").

- Opción 2, era opuesta a la opción uno, ya que no se le deba una instrucción específica, sólo se indicaba: "sigue tu propio criterio para hacerlo lo mejor posible".

No se les permitía meter dos peces en una misma pecera, si lo hacían no avanzaba el programa.

\section{Segunda fase: entrenamientos}

El objetivo de esta segunda fase fue probar los dos programas de entrenamiento: un programa vía control instruccional y el otro sin control instruccional (esto es, sólo señalando las consecuencias directas). Así la tarea se dividió en dos partes:

- Entrenamiento 1: con control instruccional (análogo experimental a la respuesta tipo pliance). El objetivo de este entrenamiento era que el sujeto aprendiera a seguir una instrucción acorde con las consecuencias determinadas en el programa: si lo hacía correctamente (es decir, siguiendo la regla dada), el participante escuchaba un sonido de aplausos y una voz que le decía "lo has hecho muy bien"; por el contrario, si cometía un error, sonaba un resorte vibrando y una voz que le decía "qué mal lo has hecho". Al principio de la fase aparecía la siguiente instrucción: "en la parte superior de la pantalla encontrarás unas instrucciones que podrás seguir y, de esa forma, conseguir el mayor número de peces vivos. Si lo haces bien, los peces vivirán.

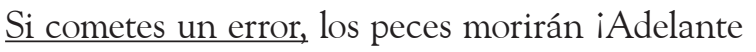
y buena suerte!".

Durante cada ensayo aparecía sólo una instrucción específica, dos peces y sus respectivas peceras (véase figura 3). Al final del entrenamiento aparecía la frase: "lo has hecho muy bien, FELICITACIO. NES, ya puedes pasar a la siguiente fase".

El criterio de éxito de los participantes estaba determinado por el número de respuestas correctas siguiendo las instrucciones dadas. Si el participan-

\section{"Instrucciones del ordenador"}
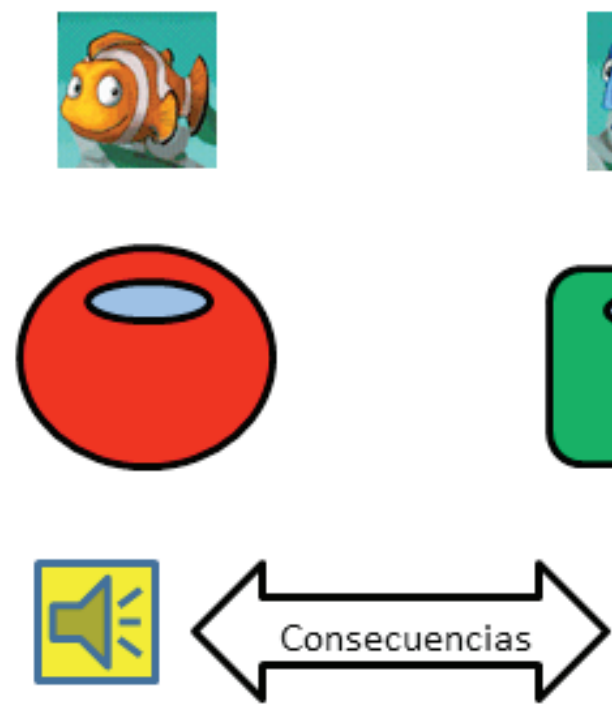
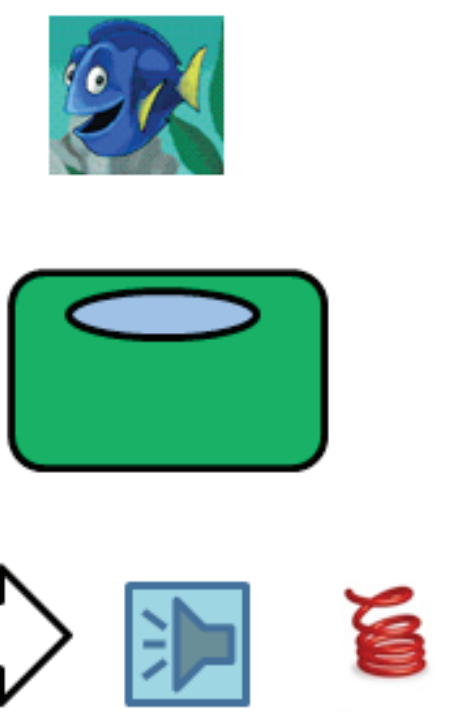

Figura 3. Ejemplo de un ensayo en la fase de reglas

Fuente: elaboración propia 
te realizaba correctamente diez ensayos seguidos, cumplía el criterio requerido para que el ordenador diera por terminada esa tarea y lo enviara directamente a la siguiente; si no cumplía este criterio, el ordenador volvía a empezar el bloque de ensayos hasta que el participante cumpliera el criterio de los diez ensayos correctos, nunca superando más de 25 (si se superaban pasaba en cualquier caso a la siguiente fase). La tarea, como tal, seguía siendo introducir cada pez en su respectiva pecera, siguiendo la instrucción dada por el ordenador y que cambiaba en cada ensayo (por ejemplo: "el pez azul debe ir en la pecera cuadrada", "el pez rojo debe ir en la pecera redonda”). Tampoco se consideraba como correcto que el participante colocara dos peces en una misma pecera.

- Entrenamiento 2: sin control instruccional, solo señalando consecuencias directas (análogo experimental a la respuesta tipo tracking). El objetivo de este entrenamiento era que el sujeto aprendiera la manera correcta de meter los peces en la pecera sin que recibiera instrucción alguna al respecto. La forma de adquirir dicho repertorio comportamental era aprenderlo sólo mediante las consecuencias directas que recibía por cada ensayo. Las consecuencias eran un sistema de puntos que a su vez fueron asociados a un refuerzo tangible: un vale regalo de un centro comercial local. Estas contingencias directas eran descritas a los participantes en las normas generales del juego: "al igual que en las partes anteriores, deberás colocar cada pez en su respectiva pecera, pero esta vez no tendrás instrucciones, el ordenador no te dirá cómo hacerlo. Tendrás que usar tu propio criterio para realizar la tarea, sigue tu instinto de pescador para encontrar la forma correcta. Tu puntaje se irá acumulando o disminuyendo a medida que avances en el juego, $\underline{\text { los puntos que obtengas en esta parte del juego los }}$ podrás canjear al final por un vale regalo".

En este tipo de entrenamiento, además de no dar al participante instrucción alguna en cada ensayo, tampoco se le daba refuerzo verbal; así mismo, el control estimular era diferente: las peceras tenían formas distintas y los colores de los peces eran diferentes, a fin de evitar que pudiesen realizar algún ensayo siguiendo el patrón de la fase anterior (fase de seguimiento instruccional). Un ensayo era considerado correcto cuando seguía el patrón marcado por el ordenador (el cual el sujeto desconocía) y que debía aprender a medida que se enfrentaba a cada ensayo. El sistema de puntos funcionaba de manera que cada vez que el niño tuviese un ensayo correcto aumentaban los peces de la pecera y el agua de la misma.

Si el participante se equivocaba (esto es, no seguía el patrón establecido por el ordenador), el nivel del agua bajaba y se veían menos peces en la pecera, a la par que el pez que había metido en la pecera equivocada se transformaba en una imagen de un esqueleto (véase figura 5). Si el niño realizaba correctamente diez ensayos seguidos, cumplía el criterio requerido para que el ordenador diera por terminada esa tarea y lo enviara directamente a la siguiente; si no cumplía este criterio, volvía a empezar el bloque de ensayos hasta que cumpliera dicho criterio, nunca superando más de 25 intentos.

En cuanto a la tarea propiamente, al igual que en las fases anteriores, el participante debía introducir cada pez en su respectiva pecera siguiendo la consecuencia que marcaba el programa, pero no se le daban instrucciones de ningún tipo durante los ensayos (véase figura 4).

Tercera fase: postest. El objetivo de esta fase fue evaluar si la tendencia inicial de los participantes a seguir instrucciones o no había cambiado después del entrenamiento en seguimiento instruccional y en moldeamiento por contingencias directas. Para determinarlo, se tuvo en cuenta el porcentaje de ensayos que el participante tenía siguiendo las reglas versus el porcentaje siguiendo contingencias. El proceder de la prueba era idéntico al pretest (sin instrucciones ni sistema de puntos).

Los datos de la tarea experimental fueron analizados por medio de un análisis descriptivo de los datos. En dicho análisis se tenía en cuenta el número de ensayos requeridos por los participantes para lograr el criterio (establecido previamente) de éxito de cada entrenamiento; en las fases del pretest y el postest, se evaluaba el total de las respuestas dadas por los participantes. Los datos del cuestionario fueron analizados agrupando los resultados de los participantes según las escalas que lo componían 


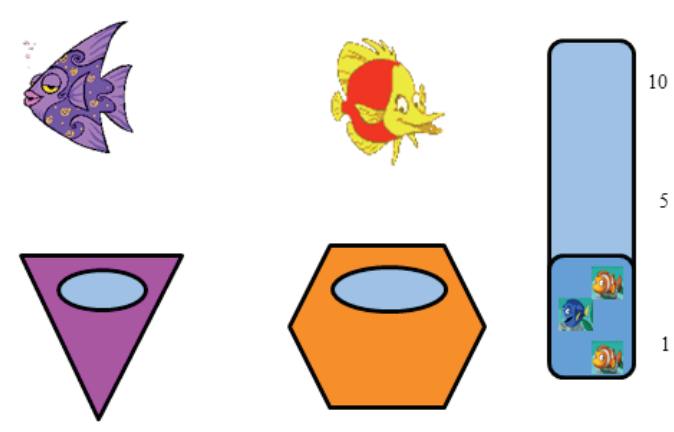

Figura 4. Ensayo fase sin control instruccional

Fuente: elaboración propia

(autocontrol, rigidez, etc.) y obteniendo los percentiles, la media y su desviación estándar.

\section{Resultados}

A continuación se presentan los resultados del estudio divididos en dos bloques. En el primer bloque se exponen los datos relativos a la tarea experimental, divididos según la tendencia que los participantes mostraron en el pretest (tendencia contraria a las instrucciones, ajustada al seguimiento instruccional o mixta) y analizando su patrón de respuesta a lo largo de las cuatro fases de la tarea (línea base, entrenamiento tipo 1 , entrenamiento tipo 2 y posttest). En el segundo bloque se presentan los resultados del cuestionario; estos se han analizado según las escalas que mide el instrumento y agrupándolos también por la tendencia inicial de los participantes en la tarea experimental. Finalmente se realiza un análisis de cómo se relacionan los puntajes del cuestionario con los resultados obtenidos por los participantes en la tarea experimental.

\section{Bloque 1: tarea experimental}

Para facilitar la exposición de los datos, estos se presentan agrupando a los participantes según su tendencia en la línea base. Esto es, "contraria a las instrucciones", "bajo control instruccional" o "mixto".

Así, en la figura 6 se muestran los resultados de los participantes 1, 2 y 3 cuya tendencia en el patrón de respuestas en línea base se estableció como "contrario a las instrucciones", ya que durante
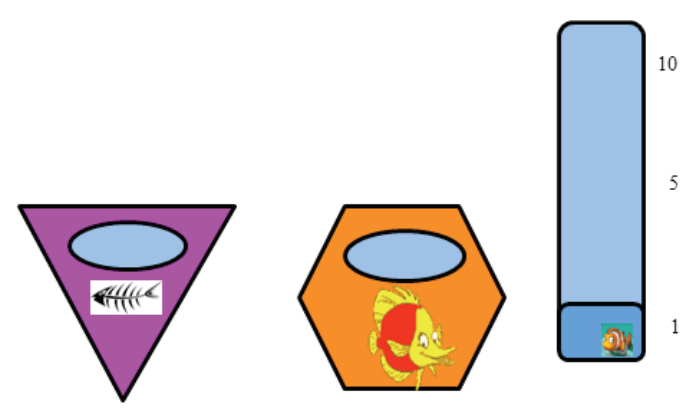

Figura 5. Ensayo donde se comete un error

Fuente: elaboración propia

los 25 ensayos sus respuestas fueron contrarias a lo indicado en la instrucción dada por el ordenador. Durante el primer entrenamiento (con control instruccional) estos tres participantes lograron cumplir el criterio para pasar a la siguiente fase en solo diez ensayos, sin tener ningún error. Durante el segundo entrenamiento (sin instrucciones, basado en contingencias directas) se muestra variabilidad en estos tres participantes: el sujeto 1 no mostró el aprendizaje del patrón deseado, tuvo un 36\% de errores que fluctuaban a lo largo de su patrón de respuesta; en cuanto al sujeto 2 , aunque tuvo un $12 \%$ de errores al principio de la fase, sí mostró haber aprendido el patrón que determinaba el programa sin volver a cometer errores, por lo cual sólo necesitó trece ensayos de los 25 que tenía disponibles; por último, el sujeto 3 tuvo un 20\% de errores distribuidos a lo largo de la fase, por lo que no parece haber aprendido el patrón establecido. En cuanto a la última fase, los sujetos 1 y 3 mostraron un mayor número de respuestas (88 y 96\%, respectivamente) siguiendo el mismo patrón que en la línea base, es decir tipo "contrario a las instrucciones"; mientras que el sujeto 2, en esta fase, mostró un cambio claro en su patrón de comportamiento, con un $84 \%$ de respuestas según el patrón de "seguir las instrucciones".

En la figura 7 se puede observar los datos de los participantes 7, 8 y 9, cuya tendencia en el patrón de respuestas de línea base se estableció como "tendencia a seguir las instrucciones", ya que durante los 25 ensayos respondieron siguiendo las reglas indicadas por el ordenador. En el primer entrenamiento los tres participantes mostraron haber 


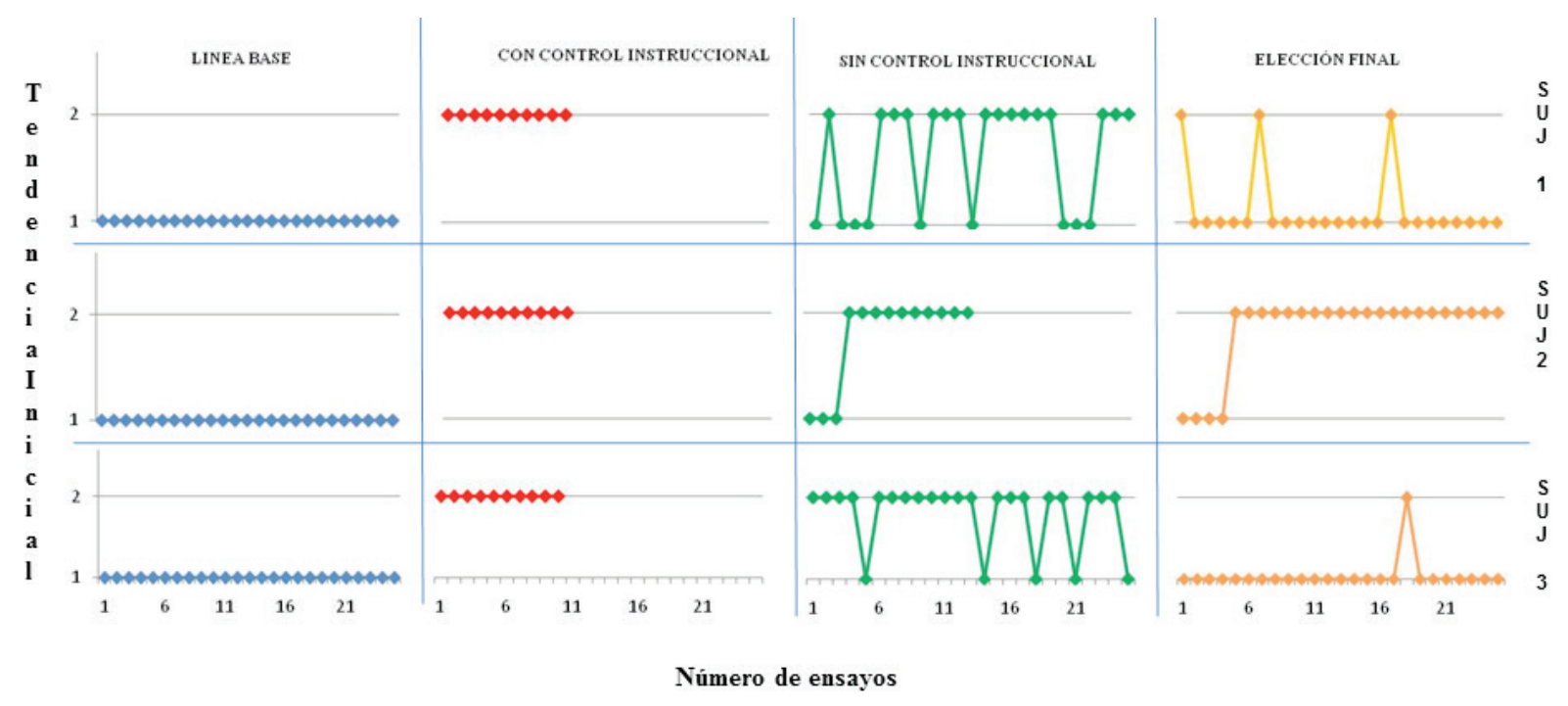

Figura 6. Grupo con tendencia inicial "contrario a las instrucciones"

Nota: en cuanto a la nomenclatura de las fases línea base y elección final, el 1 es la respuesta contraria a la instrucción y el 2 es la respuesta siguiendo la instrucción. En la fase de entrenamientos, el 1 es la respuesta incorrecta y el 2 es la respuesta correcta.

Fuente: elaboración propia

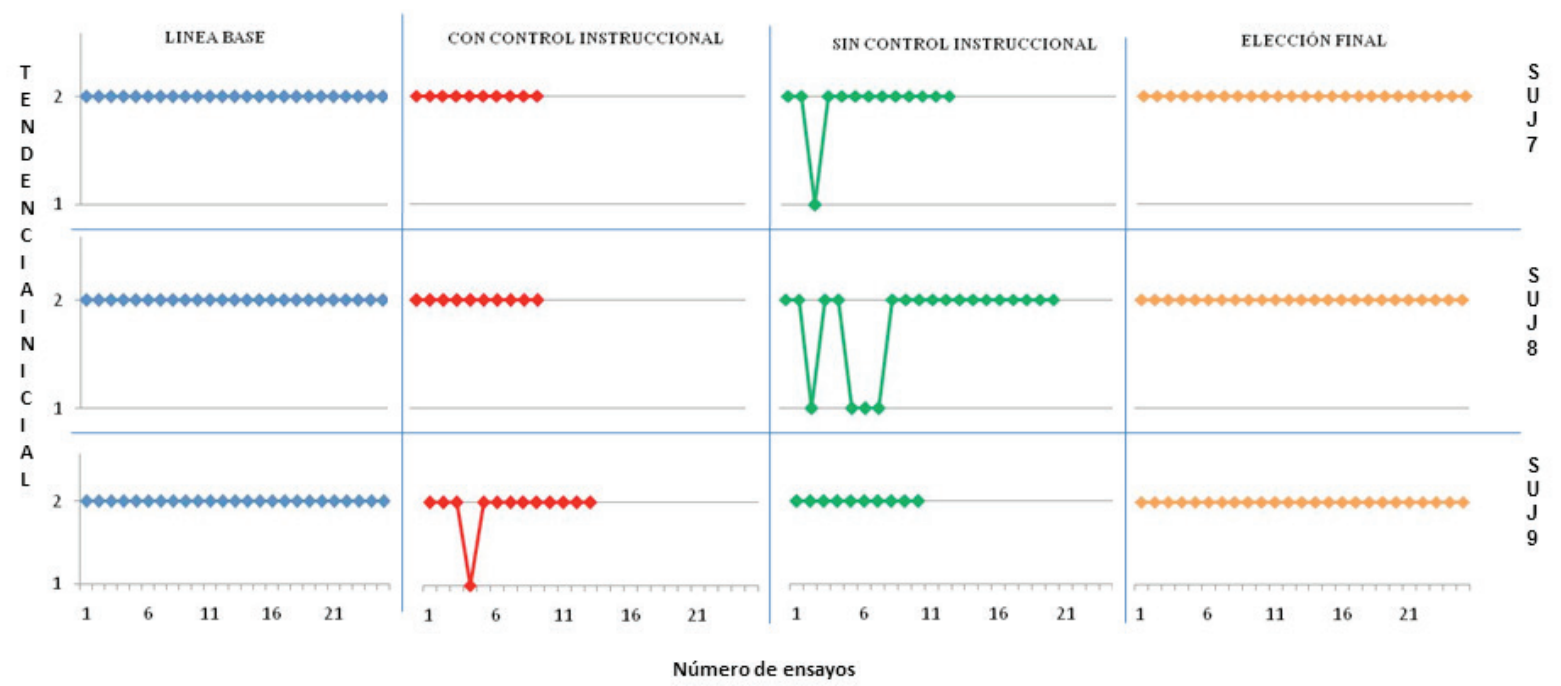

Figura 7. Grupo con tendencia inicial a "seguir las instrucciones"

Nota: en cuanto a la nomenclatura de las fases línea base y elección final, el 1 es la respuesta contraria a la instrucción y el 2 es la respuesta siguiendo la instrucción. En la fase de entrenamientos, el 1 es la respuesta incorrecta y el 2 es la respuesta correcta.

Fuente: elaboración propia

aprendido el patrón en tan solo diez ensayos, sin cometer ningún error; durante el segundo entrenamiento, los participantes 7 y 8 mostraron aprendizaje del patrón establecido (el primero a los 13 ensayos y el segundo a los 21 ensayos) con un por- centaje de error del $4 \%$ y el $16 \%$, respectivamente; mientras que el sujeto 9 no presentó ningún error y con solo diez ensayos mostró haber aprendido el patrón de respuesta correcto. Por último, en la fase final, los tres participantes mantuvieron el mismo 
patrón que en la línea base (esto es, bajo control instruccional) sin variabilidad.

La figura 8 muestra los resultados de los participantes 4,5 y 6 , con una tendencia en línea base considerada como "mixta", ya que sus respuestas fluctuaban a lo largo de toda la fase. En el primer entrenamiento los participantes 4 y 5 mostraron un patrón de respuestas estable, necesitando solo 17 ensayos el sujeto 4 , y once ensayos el sujeto 5 , con una taza de errores baja ( $12 \%$ y $4 \%$ respectivamente) y solo al principio de la ejecución; en cuanto al sujeto 6, su patrón de respuesta fue estable desde el principio y sin cometer errores, lo cual demuestra que también superó esta fase, necesitando para ello solo diez ensayos. Durante el segundo entrenamiento, el sujeto 4 tuvo un 16\% de errores antes de lograr el aprendizaje del patrón establecido, lográndolo en el ensayo número 17; el sujeto 5 presentó un patrón de respuestas fluctuante con un $18 \%$ de errores, con lo cual se confirma que no aprendió el patrón establecido; el sujeto 6 no cometió errores durante su ejecución y mostró haber aprendido el patrón en solo diez ensayos. En la última fase los participantes 4 y 5 mantuvieron el patrón fluctuante de la línea base, mientras que el sujeto 6 cambió su patrón de línea base estableciéndose como predominante sus respuestas tipo "contrario a las instrucciones".

\section{Bloque 2: cuestionario o test situacional}

En cuanto a los resultados obtenidos en el test o cuestionario creado para esta prueba, para medir aspectos potencialmente relacionados, como la rigidez, el autocontrol y la tendencia a seguir instrucciones o dejarse llevar más por las consecuencias directas, se realizó un análisis teniendo en cuenta los percentiles. A continuación se presentan los participantes que puntuaron por encima de la media en cada escala.

En cuanto a la variable autocontrol, el 56\% de los sujetos obtuvieron puntajes por debajo de la media (véase tabla 2 para los puntajes de media, desviación estándar y percentiles), particularmente el sujeto 2 se encontró por debajo de la media más una desviación, siendo el puntaje más bajo de todo el grupo en esta variable. Por el contrario, el 44\% de los sujetos se ubicaron por encima de la media, específicamente entre el percentil 75 y 95 . En la variable rigidez, el $56 \%$ de los sujetos se situó por encima de la media, entre el P75 y el P95; entre los

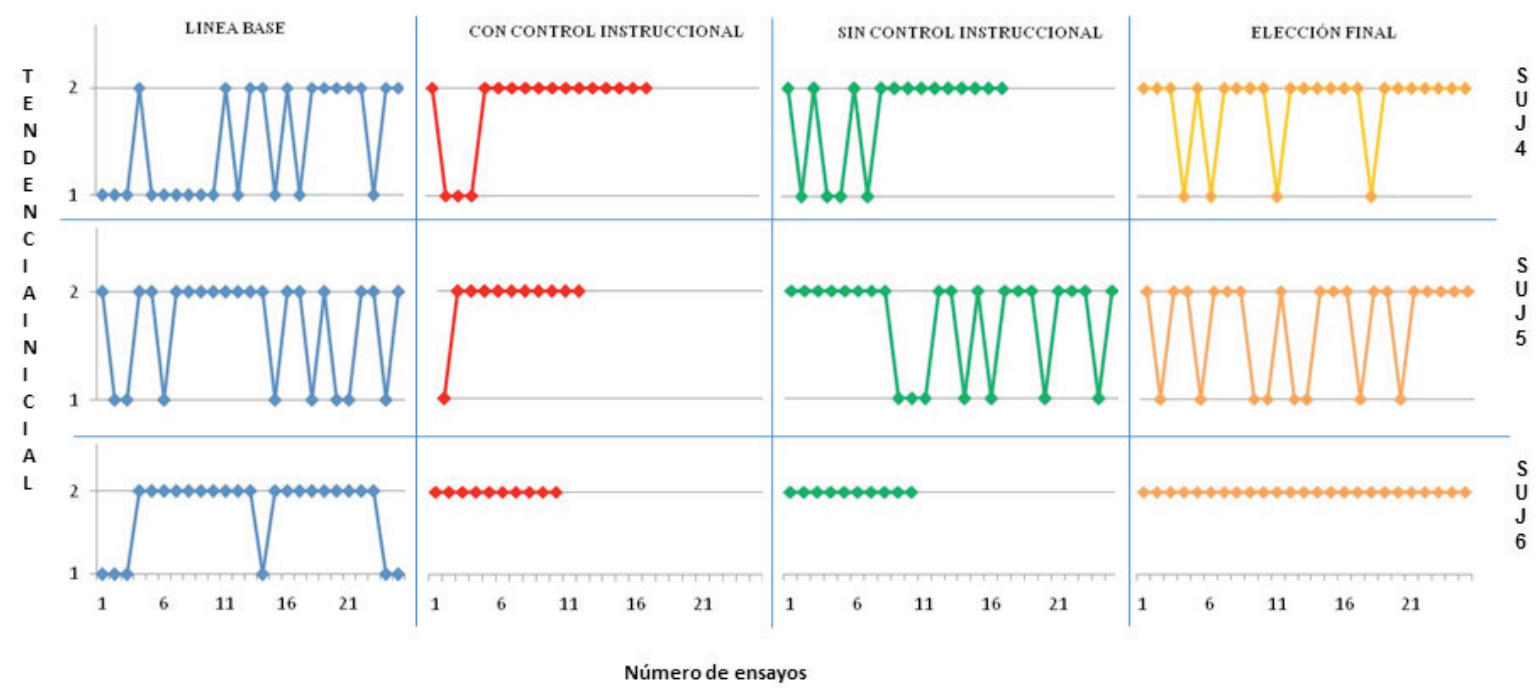

Figura 8. Grupo con tendencia inicial "mixta". Grupo con tendencia inicial a "seguir las instrucciones"

Nota: en cuanto a la nomenclatura de las fases línea base y elección final, el 1 es la respuesta contraria a la instrucción y el 2 es la respuesta siguiendo la instrucción. En la fase de entrenamientos, el 1 es la respuesta incorrecta y el 2 es la respuesta correcta.

Fuente: elaboración propia 
TABLA 2.

Puntajes de media, desviación estándar y percentiles obtenidos por el grupo en cada escala y diferenciados según si son niños o niñas

\begin{tabular}{ccccccc}
\hline & & & & \multicolumn{3}{c}{ Percentiles } \\
\cline { 5 - 7 } Niños & Variables & Media & DE & P5 & P50 & P95 \\
\cline { 5 - 7 } & Autocontrol & 6 & 1.41 & 5 & 5 & 7.8 \\
& Rigidez & 10.8 & 1.78 & 9 & 11 & 12.8 \\
& Seguimiento de reglas & 4.6 & 1.57 & 3.2 & 4 & 6.6 \\
& Tracking & 2.6 & 0.54 & 2 & 3 & 3 \\
\multirow{3}{*}{ Niñas } & Total & 24 & 1.58 & 22.2 & 24 & 25.8 \\
& Autocontrol & 6.25 & 2.21 & 4.15 & 6 & 8.7 \\
& Rigidez & 12.25 & 3.3 & 9.15 & 12 & 15.7 \\
& Seguimiento de reglas & 5.75 & 1.25 & 4.3 & 6 & 6.85 \\
& Tracking & 3.5 & 0.57 & 3 & 3.5 & 4 \\
& Total & 27.75 & 2.06 & 26 & 27.5 & 29.85 \\
\hline
\end{tabular}

Fuente: elaboración propia

puntajes por debajo de la media se encontraron el $44 \%$ de los sujetos.

En cuanto a la variable seguimiento de reglas, el $56 \%$ de los sujetos obtuvo puntajes por encima de la media, específicamente entre el P75 y el P95. Por debajo de la media se situó el 44\% de los sujetos, es llamativo el puntaje del sujeto 7 en esta variable, ya que se puntuó por debajo de la media menos una desviación, exactamente en el P5. Por último, en cuanto a la variable tracking, el 56\% de los participantes presentaron puntajes por encima de la media, situándose por encima del P75 y el 44\% de los sujetos presentaron puntajes por debajo de la media, situándose en el P25.

Al relacionar los puntajes del cuestionario con los puntajes obtenidos en el juego de ordenador o tarea experimental, se encontró que en cuanto a los participantes cuya tendencia inicial fue "contraria a las instrucciones" (véase tabla 3), el sujeto 1 presentó un puntaje alto sólo en la variable autocontrol, mientras que los participantes 2 y 3 presentaron altos puntajes en rigidez, seguimiento de reglas y tracking. En este grupo, los tres participantes tuvieron altas tasas de error al cambiar de un entrenamiento al otro, de hecho, dos de los participantes no lograron aprender el patrón del segundo entrenamiento y tampoco cambiaron su patrón de respuestas en el postest.

Por otra parte, en cuanto a los participantes con una tendencia a seguir instrucciones (véase tabla 4), los participantes 7 y 8 presentaron puntajes iguales o menores al P50, excepto en la variable tracking, en la que el sujeto 8 obtuvo un puntaje en el P75. Estos participantes tampoco presentaron dificultades en

TABLA 3.

Puntajes directos del grupo con tendencia "contrario a las instrucciones"

\begin{tabular}{lccc}
\hline Variable & \multicolumn{3}{c}{ Puntaje directo de los sujetos } \\
\cline { 2 - 4 } & Suj. 1 & Suj. 2 & Suj. 3 \\
\hline Autocontrol & 8 & 4 & 5 \\
Rigidez & 9 & 16 & 11 \\
Seguimiento de reglas & 3 & 7 & 4 \\
Tracking & 2 & 3 & 3 \\
Total & 22 & 30 & 23 \\
\hline
\end{tabular}

Fuente: elaboración propia 
el aprendizaje de ninguno de los dos entrenamientos. El sujeto 9 presentó un puntaje bastante alto en rigidez, sin embargo, tampoco tuvo dificultades para el aprendizaje de los entrenamientos, de hecho, tuvo la tasa de errores más baja en este grupo. Se podría hipotetizar que los participantes 7 y 8 presentaron puntajes normales (es decir dentro de la media) en las distintas variables medidas, lo cual se podría relacionar con la facilidad para el aprendizaje en las dos tareas. En cuanto al sujeto 9, llama la atención que, aunque obtuvo un P95 en la variable de rigidez, también presentaba altos puntajes en la variable tracking, con lo cual podría ser que esta última variable pudo influir a la hora de "probar" distintas opciones en los entrenamientos y le permitió tener muy buena ejecución. También cabría reflexionar en el hecho de que aunque las personas puedan tener una tendencia marcada en el control instruccional y puntajes altos de rigidez, no verán afectada su ejecución en el aprendizaje de nuevas tareas cuando también tienen una tendencia marcada a probar cosas nuevas y que les causan curiosidad. Así, es necesario realizar estudios en esta línea que permitan contrastar los resultados encontrados.

Por último, en cuanto a los participantes con una tendencia mixta (véase tabla 5), se encontró que el sujeto 4 presentó un puntaje alto en autocontrol y tracking y aunque tuvo una alta tasa de errores en el segundo entrenamiento, logró el aprendizaje del patrón establecido. El sujeto 5, que presentó un puntaje alto en seguimiento de reglas, no aprendió el patrón establecido en el segundo entrenamiento y mantuvo su tendencia fluctuante en el postest. El sujeto 6 obtuvo puntajes por encima del P75 en autocontrol, rigidez y seguimiento de reglas, sin que esto interfiriera en su buena ejecución a lo largo de los entrenamientos (también tuvo una de las tasas más bajas de errores durante los entrenamientos, junto con el sujeto 9), incluso llegó a cambiar su patrón de respuestas en el postest.

\section{Discusión}

Dados los resultados encontrados, se puede concluir que el entrenamiento en control instruccional que

TABla 4.

Puntajes directos del grupo con "tendencia a seguir instrucciones"

\begin{tabular}{lccc}
\hline \multirow{2}{*}{ Variable } & \multicolumn{3}{c}{ Puntaje directo de los sujetos } \\
\cline { 2 - 4 } & Suj. 7 & Suj. 8 & Suj. 9 \\
\hline Autocontrol & 5 & 7 & 5 \\
Rigidez & 14 & 9 & 13 \\
Seguimiento de reglas & 4 & 6 & 4 \\
Tracking & 3 & 4 & 3 \\
Total & 26 & 26 & 25 \\
\hline
\end{tabular}

Fuente: elaboración propia

TABla 5.

Puntajes directos del grupo con "tendencia mixta"

\begin{tabular}{lccc}
\hline \multirow{2}{*}{ Variable } & \multicolumn{3}{c}{ Puntaje directo de los sujetos } \\
\cline { 2 - 4 } & Suj. 4 & Suj. 5 & Suj. 6 \\
\hline Autocontrol & 9 & 5 & 7 \\
Rigidez & 10 & 9 & 12 \\
Seguimiento de reglas & 6 & 7 & 5 \\
Tracking & 4 & 3 & 2 \\
Total & 29 & 24 & 26 \\
\hline
\end{tabular}

Fuente: elaboración propia 
se realizó como un análogo a la regulación verbal tipo pliance, tuvo un mayor número de respuestas correctas en todos los participantes. Así mismo, el nivel de errores cometido por la muestra en general fue mucho menor en comparación con el segundo entrenamiento (sin control instruccional, sólo por consecuencias directas), lo cual indica que los participantes responden mejor al primer entrenamiento. Estos hallazgos se encuentran en un sentido contrario a lo encontrado por Ortiz, Pacheco, Bañuelos, \& Plascencia (2007), quienes estudiaron si promover el contacto con las descripciones precontacto, así como la historia de seguimiento de instrucciones, favorecen la insensibilidad al cambio contingencial respecto del grado de especificidad de las instrucciones recibidas. Sus hallazgos mostraron que hubo peores ejecuciones en aquellos participantes que en todo el experimento recibieron descripciones precontacto específicas, en contraposición con aquellos para quienes fueron de tipo genérica o para los que en el momento del cambio se encontraban recibiendo descripciones mínimas. No obstante, los hallazgos del presente estudio sí están en consonancia con lo propuesto anteriormente por Joyce \& Chase (1990), quienes mencionaban que el comportamiento moldeado directamente por contingencias puede mostrar inicialmente un alto nivel de variabilidad y una exposición relativamente larga a contingencias que puede ser necesaria antes de que se seleccione un patrón de respuesta efectiva.

Al comparar los patrones de comportamiento entre los grupos (con tendencia a seguir las instrucciones, mixto y contrario a las instrucciones) se pueden ver pautas similares de respuesta en dos de los grupos. En el grupo de tendencia a seguir las instrucciones, por ejemplo, todos los participantes mostraron haber aprendido los dos entrenamientos con una tasa de errores mínima, además de haber mantenido su patrón de respuesta inicial del pretest durante el postest. Esto apoya lo propuesto en el estudio de Wulfert, Greenway, Farkas, Hayes, \& Dougher (1994), donde se demostró que las instrucciones exactas pueden alterar el efecto de las contingencias programadas, haciendo que los sujetos perseveren más en el patrón de respuesta que habían adoptado durante la fase de refuerzo.
En el grupo contrario a las instrucciones, los participantes mostraron haber aprendido el patrón de respuesta del primer entrenamiento sin cometer errores; durante el segundo entrenamiento, dos de los participantes no aprendieron el patrón de dicho entrenamiento y tampoco cambiaron su patrón de respuesta en el postest (aunque sí tuvieron algunos ensayos contrarios a lo que mostraron en su línea base), pero el sujeto que sí logró aprender el patrón requerido del entrenamiento dos, cambió por completo su patrón en el postest. En el grupo mixto, en cambio, las fluctuaciones de respuesta también se vieron intrafase y entrefases, por ejemplo, los dos primeros participantes aprendieron el patrón del primer entrenamiento pero cometiendo una taza de errores baja, dos de los participantes no aprendieron el patrón del segundo entrenamiento y en la fase final dos de los participantes no cambiaron su patrón fluctuante.

Los resultados encontrados en el presente estudio con respecto a relacionar los puntajes obtenidos en el juego de ordenador y los del cuestionario, pese a mostrar mucha variabilidad, se encuentran en la línea de lo encontrado en el estudio de Wulfert et al. (1994), en donde se evidenció la importancia de la variable "diferencias individuales" y su efecto sobre la insensibilidad: los individuos cuyo comportamiento parecía ser menos sensible a los cambios en el programa operante, eran precisamente quienes tenían un mayor número de declaraciones que reflejaban las actitudes y comportamientos conservadores, indicativos de un gobierno, por las reglas.

Pese a que se requiere de una muestra de sujetos más amplia para sacar conclusiones definitivas a la hora de relacionar las variables establecidas en el cuestionario, con la tendencia al seguimiento de instrucciones y al aprendizaje de tareas análogas al pliance y al tracking se pudo observar que en los grupos donde las tendencias iniciales (pretest) estaban claramente establecidas, sí se existía una relación más directa entre las variables de rigidez, autocontrol y seguimiento de reglas con la facilidad o dificultad para aprender uno u otro entrenamiento. Los participantes con patrones de respuesta iniciales más fluctuantes generan nuevas inquietudes y hacen que tomemos con precaución 
la relación existente entre variables como la rigidez con la ejecución en tareas que requieren contacto directo con las instrucciones versus las tareas con contingencias directas.

De cara a futuros estudios, es importante solventar algunos aspectos por mejorar que se mencionan a continuación: se requiere una muestra más amplia que permita evaluar con mayor precisión la variabilidad intragrupos; así mismo, es necesario evaluar si el orden de presentación de los entrenamientos (primero el análogo al pliance y segundo el análogo al tracking) es una variable relevante en el aprendizaje de los mismos y en el cambio de tendencia final. Por último, es necesario evaluar con mayor profundidad y con cuestionarios de mayor validez estadística, las variables relacionadas con las diferencias individuales, se requieren ítems más discriminantes ya que el efecto mostrado es bastante relativo.

\section{Referencias}

Barnes-Holmes, D., O'Hora, D., Roche, B, Hayes, S., Bissett, R., \& Lyddy, F. (2001). Understanding and verbal regulation. In S. C. Hayes, D. BarnesHolmes, \& B. Roche (Eds.), Relational frame theory: A post-Skinnerian account of human language and cognition. (pp. 103-117) New York: Kluwer Academic/Plenum.

Dixon, M., Hayes, L., \& Aban, I. (2000). Examining the roles of rule following, reinforcement, and preexperimental histories on risk-taking behavior. The Psychological Record, 50, 687-704.

Gómez, I. (1996). Investigación sobre el fenómeno de sensibilidad - insensibilidad a las contingencias y el papel de la conducta verbal. Tesis doctoral no publicada, Universidad de Almería.

Gómez, I., Moreno, E., \& López, N. (2006). (In) Sensibilidad a unas $u$ otras contingencias en el marco de la conducta gobernada por reglas. México: Editorial Plaza y Valdés, S.A.

Gómez, I. y Luciano, C. (2000). Autocontrol a través de reglas que alteran la Función. Psicothema, 12(3), 418- 425.

Joyce, J., \& Chase, P. (1990). Effects of response variability on the sensitivity of rule- governed behavior.
Journal of the Experimental Analysis of Behavior, 54, 251-262.

Hayes, S., Barnes-Holmes, D. y Roche, B. (2001). Relational Frame Theory A Post-Skinnerian Account of Human Language and Cognition. New York: Kluwer Academic.

Hayes, S., Brownstein, A., Zettle, R., Rosenfarb, I., \& Korn, Z. (1986). Rule-Governed Behavior and Sensitivity to Changing Consequences of Responding. Journal of the Experimental Analysis of Behavior, 45(3), 237-256.

Hayes, S., Wilson, K., Gifford, E., Follette, V., \& Strosahl, K. (1996). Experiential avoidance and behavioral disorders. A functional dimensional approach to diagnosis and treatment. Journal of Consulting and Clinical Psychology, 64, 1152-1168.

Hayes, S., Gifford, E. \& Hayes, G. (1998). Moral behavior and the development of verbal regulation. The Behavior Analyst, 21(2), 253-279.

Luciano, M. (1992a). La conducta verbal a la luz de recientes investigaciones. Su papel sobre otras conductas verbales y no verbales. Psicothema, 4(2), 445-468.

Luciano, M. (1992b). Algunos significados aplicados de los tópicos de investigación básica conocidos como "relaciones de equivalencia", "decir hacer" y "sensibilidad e insensibilidad" a las contingencias. Análisis y Modificación de Conducta, 18(62), 805-859.

Luciano, M., \& Gómez-Martín, S. (2001). Derivación de funciones psicológicas. Psicothema, 13(4), 700-707.

Luciano, M., Gómez, I., \& Valdivia, S. (2002). Consideraciones acerca del desarrollo de la personalidad desde un marco funcional - contextual. International Journal of Psychology and Psychological Therapy, 2(2), 173-197.

Luciano, C., \& Valdivia, S. (2012). Nuevas perspectivas en el tratamiento de los trastornos de la personalidad. En M. Vallejo (Ed.), Manual de Terapia de Conducta, (pp.855-930). Madrid: Dykinson.

Luciano, C., Valdivia, S., \& Ruiz, F. (2012). The self as the context for rule-governed behavior. In $\mathrm{L}$. McHugh \& I. Stewart (Eds.), Self and Perspective Taking (pp.143-160). Oakland, CA: Harbinger.

Ortiz, G., Pacheco, V., Bañuelos, I., \& Plascencia, L. (2007). Efecto del contacto con instrucciones, 
la especificidad e historia instruccional en la insensibilidad al cambio contingencial en tareas de igualación de la muestra de primer orden en humanos. Acta Colombiana de Psicología, 10(2), 107-115.

Törneke, N., Luciano, C., \& Valdivia, S. (2008). Rulegoverned behavior and psychological problems. International Journal of Psychology and Psychological Therapy, 8, 141-156.
Wilson, K., \& Luciano, M. (2002). Terapia de Aceptación y Compromiso: Un Tratamientoconductual orientado a los valores. Madrid: Pirámide.

Wulfert, E., Greenway, D., Farkas, P., Hayes, S., \& Dougher, M. (1994). Correlation between selfreported rigidity and rule-governed insensitivity to operant contingencies. Journal of Applied Behavior Analysis, 27, 659-671. 
